\title{
Expanding time-temperature-transformation (TTT) diagrams to interfaces: A new approach for grain boundary engineering
}

Patrick R. Cantwella, Shuailei Mab, Stephanie A. Bojarskic, Gregory S. Rohrerc, Martin P. Harmerb*

aDepartment of Mechanical Engineering, Rose-Hulman Institute of Technology, 5500 Wabash Avenue, Terre Haute IN 47803 USA

bDepartment of Materials Science and Engineering, Center for Advanced Materials and Nanotechnology, Lehigh University, 5 East Packer Avenue, Bethlehem PA 18015 USA cDepartment of Materials Science and Engineering, Carnegie Mellon University, 5000 Forbes Avenue, Pittsburgh PA 15213 USA

*Corresponding author: Martin P. Harmer, (610) 758-4227, mph2@lehigh.edu

Co-author email addresses: Patrick R. Cantwell (cantwep@rose-hulman.edu), Shauilei Ma (mashuailei@gmail.com), Stephanie A. Bojarski (sbojarski@gmail.com), Gregory S. Rohrer (rohrer@cmu.edu)

\begin{abstract}
Bulk phase transformation kinetics were not well understood before Davenport and Bain developed time-temperature-transformation (TTT) diagrams for steel alloys in the 1930s. These powerful diagrams revolutionized the heat treatment of steel and other alloys. Grain boundaries and internal interfaces are now known to behave in a phase-like manner, referred to as 'complexions', and their transitions can be represented on TTT diagrams. We present experimental grain boundary complexion TTT diagrams for polycrystalline $\mathrm{Al}_{2} \mathrm{O}_{3}$ and $\mathrm{Y}_{2} \mathrm{O}_{3}$. Grain boundary mobility discontinuities in $\mathrm{Y}_{2} \mathrm{O}_{3}$ occur at different temperatures for different annealing times, an unusual pattern that becomes understandable when viewed on a complexion TTT diagram. Similarly, the anisotropy of $\mathrm{Al}_{2} \mathrm{O}_{3}$ complexion kinetics can be visualized with these diagrams. Complexion TTT diagrams are a graphical tool to control interface-related phenomena such as diffusion, creep, oxidation, and microstructure evolution. They could explain why two-step sintering produces dense nanocrystalline ceramics and offer insight into other processes as well.
\end{abstract}

Keywords: grain boundaries (GBs); grain growth; kinetics; grain boundary engineering; complexion 


\section{Introduction}

Controlling the kinetics of bulk phase transformations is critical to controlling the microstructure and properties of a wide variety of solid state materials. The time it takes for an isothermal phase transformation to occur can be represented on a timetemperature-transformation (TTT) diagram, which in turn can be used to prescribe successful heat treatments for a variety of materials. The TTT diagram was developed by Davenport and Bain in 1930 [1] and was a breakthrough in understanding the kinetics of bulk phase transformations. The importance and utility of the TTT diagram was immediately recognized and played a pivotal role in advancing the state of the art of steel heat treatment in particular and materials processing in general.

Internal interfaces such as grain boundaries also play a crucial role in the processing and properties of materials. Grain boundary engineering was first proposed in 1984 by Watanabe [2] and since that time has become a highly active area of research. The overarching goal of grain boundary engineering is to control the grain boundary character distribution to produce materials with better performance and properties. More recently, it has been demonstrated unequivocally that grain boundaries and other internal interfaces behave in a phase-like manner [3], undergoing transitions that can change grain boundary properties such as mobility or diffusivity by orders of magnitude. These grain boundary complexion transitions have been traditionally referred to as grain boundary 'phase' transitions [4-6]. The term 'complexion' was proposed [7] to differentiate between interfaces (e.g. grain boundaries or phase boundaries) and bulk phases, and 'complexion' is the preferred nomenclature to describe phase-like transitions at grain boundaries [8]. 
As summarized in a recent review article [8], there is a substantial body of evidence demonstrating that grain boundaries behave in a phase-like manner and that different grain boundary complexions can have dramatically different properties. Complexion transitions have been described theoretically [9] and demonstrated via computer simulations and atomistic modeling [10-12]. When a grain boundary undergoes a complexion transition, its properties such as mobility, diffusivity, and cohesive strength may change discontinuously. In particular, mobility has been shown to change by over three orders of magnitude in alumina [13]. Presumably, all grain boundary-related properties will change somewhat during a complexion transition, but not necessarily by proportional amounts. For example, it is possible that the mobility can change by three orders of magnitude while the diffusivity only changes by an order of magnitude or less. This type of disparity could be exploited in grain boundary engineering. Several equilibrium complexion diagrams have been generated [8] but the importance of the kinetic transition behavior of complexions has been overlooked thus far. Based on their phase-like behavior, it stands to reason that grain boundary complexion transitions should be able to be represented on TTT diagrams in a manner analogous to bulk phase transitions. As such, grain boundary complexion TTT diagrams have the potential to impact the field of grain boundary engineering in the same way that bulk phase TTT diagrams impacted the heat treatment of steel alloys and other materials.

In this article, we present grain boundary complexion TTT diagrams for polycrystalline yttria $\left(\mathrm{Y}_{2} \mathrm{O}_{3}\right)$ and alumina $\left(\mathrm{Al}_{2} \mathrm{O}_{3}\right)$, two ceramic materials of technological interest and importance. Alumina and yttria are often doped with impurities to influence their microstructure development and enhance their properties. For example, Eu-doped 
$\mathrm{Y}_{2} \mathrm{O}_{3}$ sinters more readily and is more optically transparent than undoped $\mathrm{Y}_{2} \mathrm{O}_{3}[14], \mathrm{Yb} / \mathrm{Er}$ codoping of $\mathrm{Y}_{2} \mathrm{O}_{3}$ influences its optical emission behavior [15], and $\mathrm{Y}$-doped $\mathrm{Al}_{2} \mathrm{O}_{3}$ has increased resistance to high-temperature creep due to yttrium segregation to grain boundaries [16]. Accordingly, the experiments used to develop the grain boundary complexion TTT diagrams presented here focus on two doped systems, Y-doped $\mathrm{Al}_{2} \mathrm{O}_{3}$ and $\mathrm{Yb} / \mathrm{Er}$ codoped $\mathrm{Y}_{2} \mathrm{O}_{3}$.

Abnormal grain growth due to a discontinuous jump in grain boundary mobility is one of the most recognizable signs of a grain boundary complexion transition in doped ceramics [8]. Atomic-resolution transmission electron microscopy has shown that grain boundaries surrounding an abnormal grain have a chemistry and structure that is distinct from boundaries of normal grains [13]. Grain growth kinetics analysis has shown that the effective grain boundary mobility of these boundaries can be over three orders of magnitude higher than grain boundaries surrounding normal grains. Therefore, by monitoring grain growth as a function of time and temperature, it should be possible to map out the conditions under which grain boundary complexion transitions occur when subjected to isothermal annealing. The grain boundary complexion TTT diagrams presented in this work were generated by analyzing grain growth behavior in Y-doped $\mathrm{Al}_{2} \mathrm{O}_{3}$ and $\mathrm{Yb} /$ Er-doped $\mathrm{Y}_{2} \mathrm{O}_{3}$ under isothermal annealing conditions.

At the outset we point out that grain boundary complexion TTT diagrams are not identically analogous to bulk phase TTT diagrams because a grain boundary has five additional macroscopic degrees of freedom that describe its character. Three degrees of freedom describe the misorientation of the two grains on either side of the boundary and two degrees of freedom describe the grain boundary plane inclination. Therefore, each 
type of grain boundary will behave differently in thermodynamic terms and have its own TTT diagram. However, the microstructure development and properties of a polycrystalline material are often dominated by a subset of grain boundary complexions that all transition within a small temperature range and thus behave in a thermodynamically similar manner [8]. The result is that in many practical situations, grain boundaries of different character can be lumped together and treated as a group when plotting diagrams.

\section{Experimental}

Two types of $\mathrm{Y}_{2} \mathrm{O}_{3}$ powders were used in the present study. Yb/Er-doped $\mathrm{Y}_{2} \mathrm{O}_{3}$ experiments were carried out with a commercially produced powder co-doped with 10 wt. $\% \mathrm{Yb}_{2} \mathrm{O}_{3}$ and 1 wt. $\% \mathrm{Er}_{2} \mathrm{O}_{3}$ or $10 \mathrm{wt} . \% \mathrm{Yb}_{2} \mathrm{O}_{3}$ and $4 \mathrm{wt} . \% \mathrm{Er}_{2} \mathrm{O}_{3}$, with a starting particle size of about $200 \mathrm{~nm}$ (Orasure Technologies, Inc., Bethlehem, PA). For the undoped experiments, a high-purity $\mathrm{Y}_{2} \mathrm{O}_{3}$ powder (H. C. Starck GmbH \& Co. KG, Goslar, Germany) was used, with a starting particle size less than $100 \mathrm{~nm}$ as determined by TEM analysis. The doped and undoped sintering specimens were produced directly from the as-received powders.

Impurities in the starting $\mathrm{Y}_{2} \mathrm{O}_{3}$ powders were quantified via inductively coupled plasma (ICP) analysis (Intertek Caleb Brett, Deer Park, TX) to determine the main impurities and their concentration. The impurities present in each powder are shown in Table 1. To avoid further contamination, the powder was uniaxially dry pressed in an alumina die under a low pressure $(<1 \mathrm{MPa})$ to form disks of $14 \mathrm{~mm}$ diameter. The disks were then rubber-bagged and cold isostatically pressed at $35 \mathrm{MPa}$ for 2 minutes. The green specimens were placed on a high purity alumina plate, covered with an alumina crucible, 
and fired in a box furnace at temperatures of $1000{ }^{\circ} \mathrm{C}$ to $1600^{\circ} \mathrm{C}$ in air. The heating and cooling rates were 6 and $5{ }^{\circ} \mathrm{C} / \mathrm{min}$, respectively. Soaking time at the sintering temperature varied from 0 to 24 hours.

The bulk density of the $\mathrm{Y}_{2} \mathrm{O}_{3}$ sintered compacts was measured by the Archimedes immersion method using distilled water with $0.01 \%$ Brij-35 as a wetting agent. Samples for microstructural examination were cut from the core of the sintered pellet to avoid surface contamination effects, polished to a $1 \mu \mathrm{m}$ finish, and thermally etched at a temperature $30^{\circ} \mathrm{C}$ below the firing temperature for 15 minutes. Heating and cooling rates for the etching treatment were $10^{\circ} \mathrm{C} / \mathrm{min}$.

The microstructure of the $\mathrm{Y}_{2} \mathrm{O}_{3}$ sintered samples was characterized by scanning electron microscopy (SEM) (Hitachi 4300; JEOL 6300F; Philips XL30 ESEM). The average grain size of each specimen was determined statistically by measuring about 1000 grains from the SEM images. Elemental analysis via energy dispersive X-ray spectroscopy (EDS) was done on a scanning transmission electron microscope (STEM) (VG Systems HB603) with a Nion Cs-corrector operating at $300 \mathrm{kV}$. X-ray diffraction (XRD) was conducted to identify the phases present in the powders and sintered specimens.

The Y-doped $\mathrm{Al}_{2} \mathrm{O}_{3}$ sandwich specimens were prepared as described elsewhere [17]. In brief, a bulk sample with (0001) and (11 $\overline{2} 0)$ single crystals of sapphire embedded in a 500 ppm Y-doped alumina polycrystal was spark plasma sintered, sectioned, and polished. Each sectioned sample was heated to a temperature between $1450{ }^{\circ} \mathrm{C}$ and $1600^{\circ} \mathrm{C}$ for times between zero and fifteen hours, where zero hours refers to heating the sample to the annealing temperature and then immediately cooling. Electron backscatter diffraction (EBSD) was used to collected orientation images of the interface and adjacent grains. While 
the authors recognize that EBSD does not give the most accurate representation of grain size, it was important to know the orientation of the single crystal along which the large grains were characterized. Using EDAX-TSL orientation imaging software (EDAX Inc., Mahwah, NJ USA), a data partition was set to remove all large grains as well as the single crystals from the scans. The average grain size of the partitioned smaller or "normal" grains was then determined. With knowledge of this value, a partition of 2.7 times the average normal grain size was used and the average normal grain size was recalculated and adjusted. This method was iterated until the partition was 2.7 times the average normal grain size. To show only larger than average grains as a metric for quantifying transitioned boundaries at the single crystal interface, a second filter was applied to the full dataset showing only grains three times larger than the previously calculated average normal grain size. In the case where there were some small grains, but the overall population of grains were clearly impinged large grains; the normal grain size cutoff was estimated by extrapolating data from the un-impinged microstructures heated for shorter times.

\section{Results}

As the experimental results presented here demonstrate, the majority of grain boundaries in the $\mathrm{Y}_{2} \mathrm{O}_{3}$ specimens behave similarly and therefore the transition kinetics for all grain boundaries in this system can be represented by a single curve on the TTT complexion diagram. On the other hand, in $\mathrm{Y}$-doped $\mathrm{Al}_{2} \mathrm{O}_{3}$, the grain boundary complexion transitions are not adequately represented by a single curve. Two different populations of grain boundaries with different grain boundary character distributions were studied in this system, one with higher average grain boundary energy and one with lower average 
energy, resulting in two curves on the TTT complexion diagram. These two curves represent the average kinetic behavior of the two different grain boundary populations.

In the $\mathrm{Y}_{2} \mathrm{O}_{3}$ experiments, grain growth kinetics were analyzed for undoped highpurity $\mathrm{Y}_{2} \mathrm{O}_{3}$ and five different commercially doped specimens with different amounts of $\mathrm{Yb}$ and Er codoping. Three of the doped specimens contained $10 \mathrm{wt} \% \mathrm{Yb}_{2} \mathrm{O}_{3}$ codoped with 1 wt $\%$, $2 \mathrm{wt} \%$, or $4 \mathrm{wt} \% \mathrm{Er}_{2} \mathrm{O}_{3}$ and two of the specimens contained $12 \% \mathrm{Yb}_{2} \mathrm{O}_{3}$ codoped with $2 \%$ or $4 \% \mathrm{Er}_{2} \mathrm{O}_{3}$. These co-doped specimens will hereafter be referred to with abbreviated notation, e.g. $10 \% \mathrm{Yb} / 1 \% \mathrm{Er}$ refers to the specimen codoped with $10 \mathrm{wt} \% \mathrm{Yb}_{2} \mathrm{O}_{3}$ and $1 \mathrm{wt} \%$ $\mathrm{Er}_{2} \mathrm{O}_{3}$. Figure 1 shows the grain size of two of the doped $\mathrm{Y}_{2} \mathrm{O}_{3}$ specimens and the undoped $\mathrm{Y}_{2} \mathrm{O}_{3}$ specimen after sintering for six hours at a series of increasing temperatures. The grain growth of undoped $\mathrm{Y}_{2} \mathrm{O}_{3}$ was smooth and continuous, with the grain size increasing gradually with temperature. In contrast to this classical behavior, the grain growth of doped $\mathrm{Y}_{2} \mathrm{O}_{3}$ showed a rapid increase in grain size at $1470{ }^{\circ} \mathrm{C}$ and $1500{ }^{\circ} \mathrm{C}$ for the $10 \%$ $\mathrm{Yb} / 1 \%$ Er-doped powder and 10\% Yb/4\% Er-doped powder, respectively. The rapid jumps in grain size at these two temperatures are the mark of a grain boundary complexion transition in which the grain boundary mobility changes discontinuously due to first-order transitions in grain boundary structure and chemistry. These complexion transitions are somewhat spread out over a small temperature range because each grain boundary has a different character and will therefore transition at a slightly different temperature [8].

The other $\mathrm{Yb} / \mathrm{Er}$ codoped $\mathrm{Y}_{2} \mathrm{O}_{3}$ specimens displayed similar jumps in grain size within this temperature range. However, no systematic correlation existed between the $\mathrm{Yb} /$ Er dopant concentration and the complexion transition temperature. Since impurities and dopants in concentrations as little as $30 \mathrm{ppm}$ are known to influence grain boundary 
complexion transitions [13], impurity analysis was carried out as shown in Table 1 . The doped $\mathrm{Y}_{2} \mathrm{O}_{3}$ specimens contained a higher concentration of $\mathrm{Al}, \mathrm{Si}$, and $\mathrm{Ca}$ impurities than the undoped $\mathrm{Y}_{2} \mathrm{O}_{3}$ powder. These impurities are suspected to be the cause of the grain boundary complexion transitions in the codoped $\mathrm{Y}_{2} \mathrm{O}_{3}$ specimens.

Table 1: Impurities concentrations determined by inductively coupled plasma mass spectrometry (ICP-MS) in the $10 \% \mathrm{Yb} / 1 \%$ Er-doped $\mathrm{Y}_{2} \mathrm{O}_{3}$ powder and the undoped $\mathrm{Y}_{2} \mathrm{O}_{3}$ powder, with units of wt. ppm $(\mathrm{mg} / \mathrm{kg})$. Doped $\mathrm{Y}_{2} \mathrm{O}_{3}$ contains more $\mathrm{Al}, \mathrm{Si}$, and $\mathrm{Ca}$ impurities than the undoped $\mathrm{Y}_{2} \mathrm{O}_{3}$.

\begin{tabular}{|c|c|c|}
\hline Element & $\mathbf{1 0 \%} \mathbf{Y b} \mathbf{1} \mathbf{1} \mathbf{0 r} \mathbf{Y}_{\mathbf{2}} \mathbf{O}_{\mathbf{3}} \mathbf{( p p m )}$ & Undoped $\mathbf{Y}_{\mathbf{2}} \mathbf{O}_{\mathbf{3}} \mathbf{( p p m )}$ \\
\hline $\mathbf{A l}$ & 123 & $<10$ \\
\hline $\mathbf{S i}$ & 118 & $<10$ \\
\hline $\mathbf{C a}$ & 62.9 & $<50$ \\
\hline $\mathbf{F e}$ & 59.5 & $<10$ \\
\hline $\mathbf{N a}$ & 31.8 & 65.2 \\
\hline $\mathbf{K}$ & 29.3 & 92.6 \\
\hline $\mathbf{M g}$ & 15.0 & $<10$ \\
\hline $\mathbf{Z n}$ & 9.6 & 14.6 \\
\hline $\mathbf{C r}$ & 5.6 & $<10$ \\
\hline $\mathbf{M n}$ & $<5.0$ & $<10$ \\
\hline
\end{tabular}

A more extensive series of experiments was carried out on $10 \% \mathrm{Yb} / 1 \%$ Er-doped $\mathrm{Y}_{2} \mathrm{O}_{3}$ to investigate the effect of sintering time on grain size and complexion transition temperature. Sintering times between zero and six hours were studied over a range of temperatures. The results of these grain growth experiments are summarized in Figure 2. The most notable result is that the complexion transition temperature appears to depend on the sintering time. The discontinuous jump in grain growth occurs at above $1500{ }^{\circ} \mathrm{C}$ for the specimen sintered for zero hours whereas the transition occurs at about $1470{ }^{\circ} \mathrm{C}$ for the specimen sintered for the longest time of six hours. In other words, the complexion transition temperature appears to depend on the annealing time. 
The reduced grain boundary mobilities of doped and undoped $\mathrm{Y}_{2} \mathrm{O}_{3}$ calculated from the grain growth experiments are plotted in Figure 3. The undoped $\mathrm{Y}_{2} \mathrm{O}_{3}$ specimen exhibits classical Arrhenius behavior, i.e. the grain boundary mobility increases continuously with increasing temperature. In contrast, both $\mathrm{Yb} /$ Er codoped $\mathrm{Y}_{2} \mathrm{O}_{3}$ specimens exhibit discontinuous jumps in grain boundary mobility, which is the signature of a grain boundary complexion transition [8].

Further analysis was carried out to determine if the Yb or Er dopants segregated to grain boundaries and if second phases were present. Scanning transmission electron microscopy (STEM) with energy-dispersive X-ray spectroscopy (EDS) showed that Yb and Er are uniformly distributed through the sintered microstructure with no preference for grain boundaries. X-ray diffraction patterns of the $10 \% \mathrm{Yb} / 1 \%$ Er-doped $\mathrm{Y}_{2} \mathrm{O}_{3}$ sintered for six hours above and below the complexion transition temperature (i.e. from 1200 to 1500 ${ }^{\circ} \mathrm{C}$ ) only exhibit diffraction peaks for cubic $\mathrm{Y}_{2} \mathrm{O}_{3}$ (PDF 08-6817). Taken together, the STEMEDS and XRD data demonstrate that the sintered $10 \% \mathrm{Yb} / 1 \%$ Er-doped $\mathrm{Y}_{2} \mathrm{O}_{3}$ specimens were comprised of a solid solution with no second phases present and no grain boundary segregation of $\mathrm{Yb}$ or Er.

To investigate the complexion transition temperature further, sintering trajectories for $10 \% \mathrm{Yb} / 1 \%$ Er-doped $\mathrm{Y}_{2} \mathrm{O}_{3}$ were measured for a series of different temperatures. The results, shown in Figure 4, demonstrate two markedly different behaviors that depend on the sintering temperature. At $1450{ }^{\circ} \mathrm{C}$, the grain size remains almost constant while density increases, demonstrating that densification essentially occurs without grain growth. At temperatures of $1470{ }^{\circ} \mathrm{C}$ and above, the trajectory is nearly vertical, i.e. grain growth proceeds without densification. The sharp change in the grain size versus density 
trajectory that occurs between 1450 and $1470{ }^{\circ} \mathrm{C}$ is unusual and in opposition to classical sintering theory. The discontinuous change in behavior at $1470{ }^{\circ} \mathrm{C}$ indicates that a grain boundary complexion transition has occurred. This is the same temperature at which the grain boundary mobility jumped discontinuously in the grain growth experiments as discussed above.

The grain growth results from the $10 \% \mathrm{Yb} / 1 \%$ Er codoped $\mathrm{Y}_{2} \mathrm{O}_{3}$ specimen from Figure 2 were replotted on time-temperature axes to produce the grain boundary complexion TTT diagram shown in Figure 5. The red circles represent points after which the discontinuous jump in grain growth occurred (i.e., large grain size) while the blue circles represent points before the transition (i.e., small grain size). The dividing line between these points shows that the grain boundary complexion transition occurs more rapidly as the temperature is increased. This visual representation of the data in Figure 2 clarifies the grain growth results and makes it simple to predict the combinations of annealing time and temperature that will produce a complexion transition, and hence will result in a very large average grain size.

Grain growth kinetics in Y-doped $\mathrm{Al}_{2} \mathrm{O}_{3}$ were investigated using a novel sandwichstyle specimen in which 500 ppm Y-doped $\mathrm{Al}_{2} \mathrm{O}_{3}$ powder was spark plasma sintered (SPS) between two single crystal $\mathrm{Al}_{2} \mathrm{O}_{3}$ wafers. Details of the specimen preparation process [17] and relevant results from experiments in this materials system have been published previously $[17,18]$ and will be briefly explained here. However, the experimental data that demonstrates the time-temperature correlation of the complexion transitions in Ydoped $\mathrm{Al}_{2} \mathrm{O}_{3}$ is presented here for the first time. 
The orientations of the single crystals in the sandwich-style Y-doped $\mathrm{Al}_{2} \mathrm{O}_{3}$ specimen were selected so that one crystal had its C-plane (0001) facing the powder and the other crystal had its A-plane (112̄0) facing the powder. These two crystal planes were selected based on the results of grain boundary character distribution measurements that demonstrated that relatively few grain boundaries contained (0001) planes after annealing at $1600^{\circ} \mathrm{C}$, while a relatively large number of boundaries contained $(11 \overline{2} 0)$ planes [18]. These results demonstrate that grain boundaries containing (0001) planes have a higher

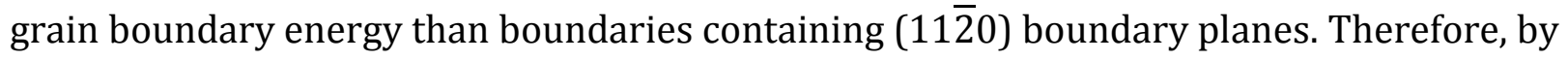
producing a sandwich specimen in which one single crystal of each plane orientation exists, the population of grain boundary characters can be biased toward low energy boundaries or high energy boundaries along the (1120) or (0001) single crystal boundaries, respectively. To confirm that the sandwich specimen successfully biased the grain boundary energies along each single crystal interface, the relative grain boundary energies were measured by atomic force microscopy (AFM), which showed that grain boundary energies along the A-plane crystal interface were lower on average than grain boundaries far from the single crystal, and that grain boundary energies along the C-plane were higher on average than grain boundaries far from the crystal [17].

The percentage of A-plane or C-plane boundary that transitioned from the low temperature to high temperature complexion was calculated by measuring the length of abnormal grain boundaries along the single crystal interfaces. At each combination of time, temperature, and interface type (A- or C-plane interface) an interface length of about 1000 $\mu \mathrm{m}$ was analyzed. Depending on the annealing conditions, average grain sizes of normal grains ranged from approximately 1 to $5 \mu \mathrm{m}$ while average sizes of abnormal grains ranged 
from 5 to $15 \mu \mathrm{m}$. These measurements were carried out on electron backscatter diffraction (EBSD) maps. The measurement method is shown graphically in Figure 6. While this straight-line measurement method produces distances that are shorter than the true, meandering interface distance, all interfaces were treated in the same way and any related uncertainty likely affected all measurements. In the case that the single crystal interface had advanced much farther than a few grain sizes into the matrix, multiple lines were combined to determine the total length of the interface, as shown in Figure 6(d). Lines were then manually drawn between triple points of abnormal grains that were connected to each interface. The total length of all of the boundaries between an abnormal grain and the single crystal was divided by the total length of the single crystal to determine a percent interface transformed. This method worked well in the cases where there was a clear bimodal distribution. However, after the large grains began to impinge on each other leading to a normal distribution with a much larger grain size, it was evident that even with the extrapolated normal grain size, an accurate representation of impinged abnormal grains could not be determined.

The results of the percentage transformation are displayed in bar graph format in Figure 7. One overall trend visible in Figure 7 is that grain boundary complexion transitions occur at lower temperatures and shorter annealing times along the high-energy C-plane (0001) single crystal interface than they do along the low-energy A-plane (11 $\overline{2} 0)$ interface. Another trend is that a higher percentage of grain boundary is transformed at longer annealing times along both single crystal interfaces. Two outliers exist in this data for the samples annealed for 2 hours at $1500^{\circ} \mathrm{C}$ and 0.5 hours at $1600{ }^{\circ} \mathrm{C}$. It is believed that these outliers exist due to the distribution of grain boundary energies along each single 
crystal interface [17] and the stochastic nature of the measurement process which does not necessarily represent all grain boundary characters with equal probability. These outliers are accordingly not included in subsequent analysis because they do not represent the overall trends evident in the data.

When the numerical data from Figure 7 are plotted on a logarithmic time scale it can be seen that their trajectory conforms to Avrami transformation behavior as shown in Figure 9. The Avrami equation is often used to analyze experimental bulk phase transformation kinetics $[19,20]$. Although it strictly only applies to bulk transformations, to maintain the analogy between bulk phase kinetics and complexion kinetics, we will apply the Avrami equation to describe the grain boundary complexion transition kinetics:

$$
y=1-\exp \left(-k t^{n}\right)
$$

where $y$ is the fraction of grain boundaries that have undergone a complexion transition at time $t, k$ is the rate constant, $n$ is the Avrami exponent, and $k$ and $n$ are assumed to be timeindependent constants. As mentioned, applying the Avrami equation to complexion kinetics is not necessarily theoretically justified, so we present the following analysis cautiously and with the primary goal of developing a complexion TTT diagram that illustrates the overall complexion kinetics present in the experimental data. To that end, the Avrami constants $k$ and $n$ were determined. These parameters are often determined by rearranging Eq. (1) into a linear form:

$$
\ln (-\ln (1-y))=\ln (k)+n \ln (t)
$$

The values of $n$ and $k$ can then be determined from the slope and intercept of the line produced by Eq. (2) that best fits the experimental data points. This fitting process is 
illustrated in Figure 8 for the data at $1600^{\circ} \mathrm{C}$ and the Avrami parameters determined in this manner are summarized in Table 2.

Table 2: Avrami constants $\boldsymbol{k}$ and $\boldsymbol{n}$ determined by fitting experimental data to the linearized Avrami equation, and the activation energy for the transitions along the A-plane and C-plane determined by fitting the rate constants $k$ to an Arrhenius equation.

\begin{tabular}{|c|c|c|c|}
\hline & $k$ & $n$ & $E_{A}$ \\
\cline { 1 - 3 } A-plane, $1500^{\circ} \mathrm{C}$ & $0.0356 \mathrm{hr}^{-0.86}$ & 0.86 & \multirow{2}{*}{$2.00 \mathrm{eV}$} \\
\cline { 1 - 3 } A-plane, $1600^{\circ} \mathrm{C}$ & $0.0715 \mathrm{hr}^{-0.95}$ & 0.95 & \\
\cline { 1 - 3 } C-plane, $1500^{\circ} \mathrm{C}$ & $0.2685 \mathrm{hr}^{-0.36}$ & 0.36 & \multirow{2}{*}{$1.57 \mathrm{eV}$} \\
\hline C-plane, $1600^{\circ} \mathrm{C}$ & $0.4650 \mathrm{hr}^{-0.42}$ & 0.42 & \\
\hline
\end{tabular}

The activation energies for the complexion transitions along the A-plane and Cplane were determined by fitting the data for the rate constant, $k$, in Table 2 to the equation, $k=k_{0} \exp \left(\frac{E_{A}}{k_{B} T}\right)$, where $E_{A}$ is the activation energy, $k_{B}$ is Boltzmann's constant, $T$ is temperature. The activation energies for the A-plane and C-plane were found to be 2.00 and $1.57 \mathrm{eV}$, respectively, as shown in Table 2 . We note here that these activation energies have been calculated for the sake of completeness, but should not be relied upon for mechanistic interpretations. First, they were calculated based on data from only two temperatures and second, they are subject to the same experimental errors as the Avrami rate constant, $k$, which will be addressed below.

The values of $k$ and $n$ shown in Table 2 were used to extrapolate the experimental data so that the times at which $10 \%, 50 \%$, and $90 \%$ of grain boundaries would undergo a complexion transition at $1500{ }^{\circ} \mathrm{C}$ and $1600^{\circ} \mathrm{C}$ could be estimated. These six transformation times, plotted as six discrete points in Figure 10, were calculated by rearranging Eq. (1) and solving for $t$ at a given fraction $y$, i.e. $y=0.1,0.5$, or 0.9 . Curves were fit to these points to produce the grain boundary complexion TTT diagram for Y- 
doped $\mathrm{Al}_{2} \mathrm{O}_{3}$, which is shown in Figure 10. The curve fits were obtained with the exponential function $T=A \exp \left(B t^{-D}\right)+T_{C}$, where $A, B$, and $D$ are fitting parameters and $T_{C}$ is the complexion transition temperature. Because they are derived from a simple exponential function, these curves are not meant to be rigorously correct from a thermodynamic point of view, rather they are intended to be visual guides to show the overall transformation kinetics from the experiments. This complexion TTT diagram is a visual representation of the kinetics data shown in Figure 7 and Figure 9. As discussed below, this data suggests that grain boundaries with higher energy will transition more quickly and at lower temperatures than boundaries with lower energy. Based on this implication, the complexion transition temperature $\mathrm{T}_{\mathrm{C}}$ for the A-plane interface is depicted in Figure 10 as being at a slightly higher temperature of $1435^{\circ} \mathrm{C}$ than the C-plane interface, whose $\mathrm{T}_{\mathrm{C}}$ is shown as $1425^{\circ} \mathrm{C}$. A transition temperature of $1425^{\circ} \mathrm{C}$ was chosen because from preliminary experiments, it was found that no grain boundaries underwent a transition at this temperature, even for long times, whereas transitions occurred at $1450{ }^{\circ} \mathrm{C}$ for the relatively long annealing time of 15 hours, as shown in Figure 7. It is apparent from this complexion TTT diagram that grain boundaries along the higher energy C-plane interface undergo a complexion transition at earlier times than those along the lower energy A-plane interface, and therefore that grain boundary anisotropy plays a significant role in complexion transition kinetics.

\section{Discussion}

The experimental data presented here demonstrate that grain boundary complexion transitions have a time dependence and that their transition kinetics can be represented on 
complexion TTT diagrams in a manner analogous to bulk phase TTT diagrams. These complexion TTT diagrams suggest that grain boundary complexion transitions propagate by a nucleation and growth method. There is a time delay during which the complexions transitions nucleate, and then a growth period during which they spread along the grain boundary and throughout the microstructure.

There are some modifications to the analogy between complexion TTT diagrams and bulk phase TTT diagrams that should be made when interpreting the results of this study. First, the complexion TTT diagrams presented here represent the transition from a low temperature complexion to a high temperature complexion during isothermal heating. In contrast, bulk phase TTT diagrams usually represent the transition from a high temperature phase to a low temperature phase during isothermal cooling. A "nose" does not exist in the complexion TTT diagrams because they are isothermal heating diagrams rather than isothermal cooling diagrams. Accordingly the transition rate increases with increasing temperature and does not reach a maximum at an intermediate temperature. Isothermal heating conditions were used to produce the complexion TTT diagrams because many grain boundary-related phenomena, especially degradation processes of technological importance, occur upon heating, e.g. creep, oxidation, and abnormal grain growth. Although the reverse transition upon cooling could also be shown on a complexion TTT diagram, the heating transition is often of greater interest in applications.

Although grain growth discontinuities are an accepted marker of grain boundary complexion transitions [8], the appearance of larger grains due to a discontinuous jump in mobility will lag behind the transition of the grain boundary itself. In other words, the complexion TTT diagrams in Figure 5 and Figure 10 include not only the time required for 
the complexion transition to occur, but also the time required for the grains adjacent to the transitioned boundaries to grow large enough to be experimentally observed as abnormally large. Despite this experimental issue, the times depicted in the grain boundary complexion TTT diagram appear to be reasonable when compared to the time required for surface phase transitions to occur. In the case of the Si (111) surface, this transition is on the order of tens of seconds to a few minutes at experimentally investigated temperatures [19]. To a first approximation, grain boundary transition times should require a similar amount of time to occur as surface phase transitions, which is generally true based on the grain boundary complexion TTT diagrams presented here. It is important to keep in mind that the time required to transition is a strong function of temperature and will change by orders of magnitude as the temperature is changed. In addition to the above differences between complexion and bulk phase TTT diagrams, grain boundaries have five additional macroscopic degrees of freedom when compared to bulk phases. Each different grain boundary character should in principle have its own curve on the complexion TTT diagram. In practice, since many grain boundaries transition under similar conditions [8], these multiple curves can be collapsed onto one or two curves as was done here for the complexion TTT diagrams for $\mathrm{Y}_{2} \mathrm{O}_{3}$ and $\mathrm{Al}_{2} \mathrm{O}_{3}$, respectively. Even though the effective grain boundary mobility in $\mathrm{Y}_{2} \mathrm{O}_{3}$ underwent a discontinuous jump during the complexion transition as shown in Figure 3, abnormal grain growth did not occur in this system. Instead, there was normal grain growth and a unimodal grain size distribution above and below the complexion transition temperature, with the average grain size increasing dramatically over a small temperature range of about $20^{\circ} \mathrm{C}$. From these observations it can be inferred that many grain boundaries in this 
system transition under very similar conditions and a single curve on the complexion TTT diagram is an appropriate way to describe their transition kinetics collectively.

In contrast, abnormal grain growth and a bimodal grain size distribution was observed in the $\mathrm{Y}$-doped $\mathrm{Al}_{2} \mathrm{O}_{3}$ specimens between 1450 and $1600^{\circ} \mathrm{C}$, while above $1600{ }^{\circ} \mathrm{C}$ and below $1450{ }^{\circ} \mathrm{C}$ the grain size distribution was normal. The abnormal grain growth in this $150^{\circ} \mathrm{C}$ temperature range demonstrates that two populations of grain boundaries underwent complexion transitions at markedly different times and temperatures. This observation was the reason for creating the sandwich style specimen so that two different populations of grain boundaries could be measured, one with higher average energy (Cplane) and one with lower average energy (A-plane).

Similarly, the $\mathrm{Y}_{2} \mathrm{O}_{3}$ complexion TTT diagram only has a single transition curve rather than start and end curves, whereas the $\mathrm{Al}_{2} \mathrm{O}_{3}$ complexion TTT diagram has curves marking the start and end of the complexion transitions. The abnormal grain growth that occurred in the Y-doped $\mathrm{Al}_{2} \mathrm{O}_{3}$ specimens enabled these start and end curves to be plotted because the percentage of interface transformed could be measured based on the abnormal grain coverage. In contrast, since abnormal grain growth did not occur in $\mathrm{Y}_{2} \mathrm{O}_{3}$, there was no range of times over which the different stages of complexion transition could be tracked, and therefore no start and end curves are depicted even though they should in principle exist.

The experimentally fitted Avrami curves from Figure 9 were used to plot the start and end transition curves in the $\mathrm{Al}_{2} \mathrm{O}_{3}$ complexion TTT diagram in Figure 10. The Avrami curves fit the data for the A-plane interface transitions reasonably well at both 1500 and $1600^{\circ} \mathrm{C}$. However, the Avrami curves do not fit the data points for the C-plane transitions 
at $1600{ }^{\circ} \mathrm{C}$ as well. One potential reason for this poorer fit is that the distribution of grain boundary energies and hence grain boundary character along the C-plane interface is much wider than along the A-plane interface [17]. Since each type of grain boundary character will require a different amount of time to transition at the same temperature, the C-plane transition is expected to be more spread out in time than the A-plane transition and hence not fit as well.

The relatively poor counting statistics of the experiment also contribute to the less than perfect fit of the data points to the Avrami equation. Even though $1000 \mu \mathrm{m}$ of interface length was examined for each combination of time and temperature and interface type (A or C interface), totaling over 20,000 $\mu \mathrm{m}$ of interface length examined, the transformation kinetics depend on the observation of abnormal grains along this interface. For shorter annealing times, abnormal grains are a relatively rare event, and as time progresses it becomes difficult to differentiate abnormal grains from normal grains. This tends to make measurements at short time prone to statistical fluctuations, while measurements at longer times tend to be less robust. The Avrami exponents presented in Table 2 are the first Avrami exponents measured for complexion transitions and, while the value of the exponent is sometimes associated with the nucleation mechanism for a bulk transformation, the significance is currently not obvious for a 2D complexion transition.

Because the $\mathrm{Yb} / \mathrm{Er}$-doped yttria specimens in the present experiment are not quite fully dense, but only nearly fully dense, it is important to consider the effect of porosity on the grain growth data. The density of the $10 \% \mathrm{Yb} / 1 \%$ Er-doped specimens was approximately 97 to $98 \%$ between the temperatures of 1450 and $1480{ }^{\circ} \mathrm{C}$, the temperature range over which the complexion transition occurred. Therefore, the maximum difference 
in porosity at the temperatures below and above the complexion transition is only about $1 \%$. This difference in porosity is too small to explain the mobility discontinuity, which is almost two orders of magnitude. The same difference in porosity exists for the undoped specimen, which does not undergo a mobility discontinuity. Furthermore, it has been shown that grain growth in $\mathrm{Y}_{2} \mathrm{O}_{3}$ is controlled by grain boundaries and that pore drag is negligible [20]. Therefore, the evidence suggests that porosity does not play a role in the observed grain boundary mobility discontinuity.

Finally, it is important to mention that the outer layer of the Yb/Er-doped yttria sintered pellets in this study had a much smaller grain size than the material at the center of the pellets. The fine grain size on the outer layer of the pellets is probably due to impurities that enter the specimen from the furnace. Although the impurities and their concentration were not identified in this study (the outer layer was removed to enable accurate grain size analysis), the presence of this fine-grained microstructure suggests that certain impurities may be very beneficial for inhibiting grain growth in $\mathrm{Y}_{2} \mathrm{O}_{3}$ and achieving a dense nanocrystalline microstructure.

One potential application of grain boundary complexion TTT diagrams is the production of bulk nanocrystalline metals and ceramics. For example, the complexion TTT diagrams presented here can potentially explain the underlying mechanism responsible for the success of two-step sintering. Two-step sintering is a method developed by Chen and Wang [21] for producing dense, fine-grained ceramics by first firing at a high temperature and then at a lower temperature. They produced near fully dense yttria $\left(\mathrm{Y}_{2} \mathrm{O}_{3}\right)$ with a grain size of $60 \mathrm{~nm}$ using $1 \% \mathrm{Mg}$ doping. In two-step sintering, grain growth is suppressed at the lower temperature while diffusivity remains high enough to enable densification. The 
underlying mechanism responsible for the success of two-step sintering is not understood, but the complexion TTT diagrams shown here could offer insight into the process. It is possible that two-step sintering is successful when the first high-temperature firing step is short enough to avoid a complexion transition - and hence to avoid runaway grain growth

- while the second sintering step is at a temperature below the complexion transition temperature. In this way the high temperature firing step could provide enhanced kinetics for accelerated densification while the low temperature firing enables densification to proceed while maintaining much slower grain growth associated with the low temperature grain boundary complexion.

\section{Conclusions}

Complexion TTT diagrams can provide precise control over the nucleation and growth kinetics of grain boundary transitions. This new tool to control grain boundary structure and properties has the potential to impact the field of grain boundary engineering as well as important applications in which grain boundary properties can be dominant, such as high temperature oxide scale growth and rare earth element doping for creep resistance in high temperature ceramics. Future studies that examine the effect of grain boundary character on transition time will be important in furthering the study of complexion transition kinetics. Additionally, the development of new techniques to more directly probe the time dependence of the complexion transitions and their associated properties discontinuities, rather than relying on microstructural markers such as abnormal grain growth, will be of great use in developing complexion TTT diagrams. In particular, studies that decouple complexion transition kinetics from grain growth kinetics will be of great value to the field. Given the difficulty and complexity of studying internal 
interfaces such as grain boundaries, it is likely that a suite of advanced characterization techniques will be needed to develop more accurate and powerful grain boundary complexion TTT diagrams. These efforts could be rewarded with a variety of advances in the field of grain boundary engineering.

\section{Acknowledgements}

The authors are grateful for support from the NSF (grant no. DMI-0457602), the Pennsylvania Department of Community and Economic Development (contract no. C000007361), the ONR-MURI program (grant no. N00014-11-0678), and the U.S. DOE

Office of Basic Energy Science Grants in the Electron and Scanning Probe Microscopies Program (grant No. DE-FG02-08ER46511). The authors are also grateful to Jeffrey M. Rickman for helpful discussions regarding data analysis.

\section{References}

[1] E.S. Davenport, E.C. Bain, Transformation of austenite at constant subcritical temperatures, Trans. AIME. 90 (1930) 117-144.

[2] T. Watanabe, An approach to grain boundary design of strong and ductile polycrystals, Res. Mech. 11 (1984) 47-84.

[3] M.P. Harmer, The phase behavior of interfaces, Science. 332 (2011) 182-183. doi:10.1126/science.1204204.

[4] J.W. Cahn, Transitions and phase equilibria among grain boundary structures, J. Phys. Colloques. 43 (1982) C6-199-C6-213. doi:10.1051/jphyscol:1982619.

[5] E.W. Hart, Grain Boundary Phase Transformations, in: H. Hu (Ed.), The Nature and Behavior of Grain Boundaries, Plenum Press, 1972: pp. 155-170. 
[6] E.W. Hart, Two-dimensional phase transformation in grain boundaries, Scripta Metall. 2 (1968) 179-182. doi:10.1016/0036-9748(68)90222-6.

[7] M. Tang, W.C. Carter, R.M. Cannon, Diffuse interface model for structural transitions of grain boundaries, Phys. Rev. B. 73 (2006) 024102. doi:10.1103/PhysRevB.73.024102.

[8] P.R. Cantwell, M. Tang, S.J. Dillon, J. Luo, G.S. Rohrer, M.P. Harmer, Grain boundary complexions, Acta Mater. 62 (2014) 1-48. doi:10.1016/j.actamat.2013.07.037.

[9] T. Frolov, Y. Mishin, Phases, phase equilibria, and phase rules in low-dimensional systems, J. Chem. Phys. 143 (2015) 044706. doi:10.1063/1.4927414.

[10] T. Frolov, D.L. Olmsted, M. Asta, Y. Mishin, Structural phase transformations in metallic grain boundaries, Nat Commun. 4 (2013) 1899. doi:10.1038/ncomms2919.

[11] T. Frolov, S.V. Divinski, M. Asta, Y. Mishin, Effect of interface phase transformations on diffusion and segregation in high-angle grain boundaries, Phys. Rev. Lett. 110 (2013) 255502. doi:10.1103/PhysRevLett.110.255502.

[12] T. Frolov, M. Asta, Y. Mishin, Segregation-induced phase transformations in grain boundaries, Phys. Rev. B. 92 (2015) 020103. doi:10.1103/PhysRevB.92.020103.

[13] S.J. Dillon, M. Tang, W.C. Carter, M.P. Harmer, Complexion: A new concept for kinetic engineering in materials science, Acta Mater. 55 (2007) 6208-6218. doi:10.1016/j.actamat.2007.07.029.

[14] S.R. Podowitz, R. Gaumé, R.S. Feigelson, Effect of europium concentration on densification of transparent Eu:Y2O3 scintillator ceramics using hot pressing, J. Am. Ceram. Soc. 93 (2010) 82-88. doi:10.1111/j.1551-2916.2009.03350.x. 
[15] F. Vetrone, J.C. Boyer, J.A. Capobianco, A. Speghini, M. Bettinelli, Effect of Yb3+ codoping on the upconversion emission in nanocrystalline Y203:Er3+, J. Phys. Chem. B. 107 (2003) 1107-1112. doi:10.1021/jp0218692.

[16] J.P. Buban, K. Matsunaga, J. Chen, N. Shibata, W.Y. Ching, T. Yamamoto, et al., Grain boundary strengthening in alumina by rare earth impurities, Science. 311 (2006) 212215. doi:10.1126/science.1119839.

[17] S.A. Bojarski, M.P. Harmer, G.S. Rohrer, Influence of grain boundary energy on the nucleation of complexion transitions, Scripta Mater. 88 (2014) 1-4. doi:10.1016/j.scriptamat.2014.06.016.

[18] S.A. Bojarski, M. Stuer, Z. Zhao, P. Bowen, G.S. Rohrer, Influence of Y and La additions on grain growth and the grain-boundary character distribution of alumina, J. Am. Ceram. Soc. 97 (2014) 622-630. doi:10.1111/jace.12669.

[19] J.B. Hannon, H. Hibino, N.C. Bartelt, B.S. Swartzentruber, T. Ogino, G.L. Kellogg, Dynamics of the silicon (111) surface phase transition, Nature. 405 (2000) 552-554. doi:10.1038/35014569.

[20] G. Bernard-Granger, N. Monchalin, C. Guizard, Sintering of ceramic powders: Determination of the densification and grain growth mechanisms from the "grain size/relative density" trajectory, Scripta Materialia. 57 (2007) 137-140. doi:10.1016/j.scriptamat.2007.03.030.

[21] I. W. Chen, X. H. Wang, Sintering dense nanocrystalline ceramics without final-stage grain growth, Nature. 404 (2000) 168-171. 


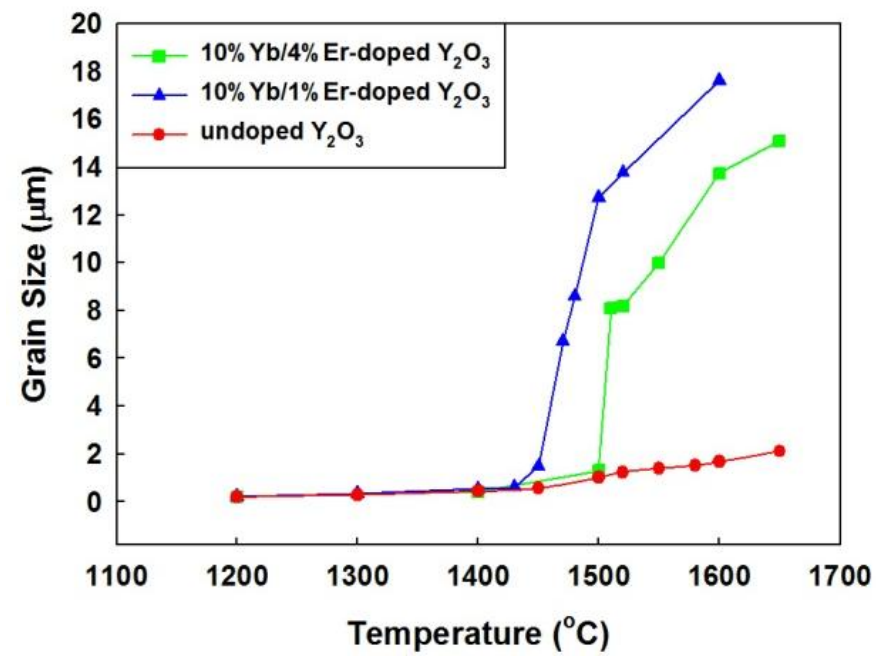

Figure 1: Effect of sintering temperature on grain size for $\mathrm{Yb} / \mathrm{Er}$-doped $\mathrm{Y}_{2} \mathrm{O}_{3}$ and undoped $\mathrm{Y}_{2} \mathrm{O}_{3}$ sintered for 6 hours. The grain size trajectory of $\mathrm{Yb} / \mathrm{Er}$-doped $\mathrm{Y}_{2} \mathrm{O}_{3}$ increases sharply at about 1470 and $1500{ }^{\circ} \mathrm{C}$, whereas the undoped $\mathrm{Y}_{2} \mathrm{O}_{3}$ grain growth increases steadily. Sintering time was 6 hours for all data points.

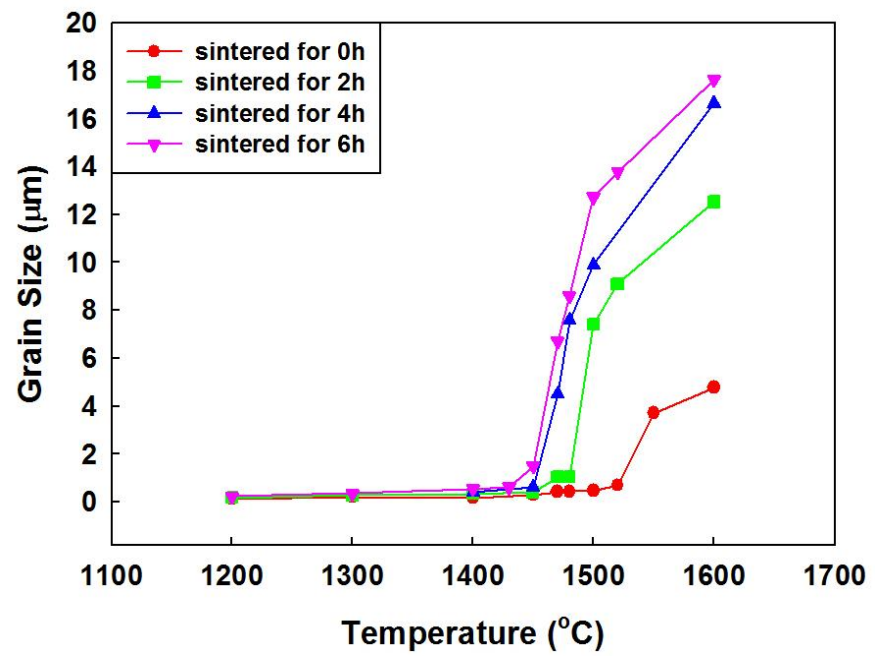

Figure 2: The effect of sintering time on grain size for different sintering temperatures in $10 \% \mathrm{Yb} / 1 \% \mathrm{Er}$-doped $\mathrm{Y}_{2} \mathrm{O}_{3}$. The grain boundary complexion transition temperature is higher for shorter sintering times and lower for longer times. 


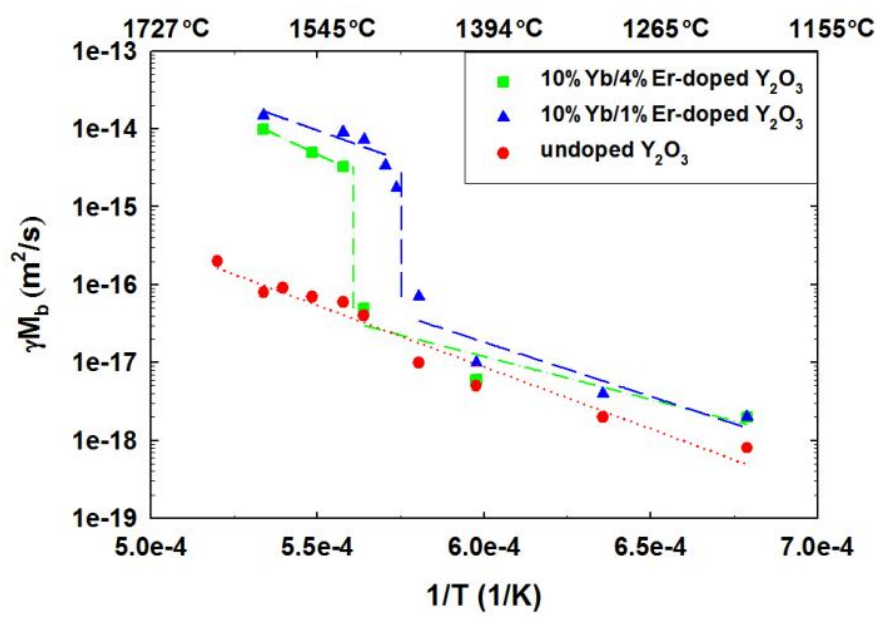

Figure 3: Reduced grain boundary mobility versus sintering temperature for $\mathrm{Yb} / \mathrm{Er}$-doped $\mathrm{Y}_{2} \mathrm{O}_{3}$ and undoped $\mathrm{Y}_{2} \mathrm{O}_{3}$. The grain boundary mobility of $\mathrm{Yb} / \mathrm{Er}$ doped $\mathrm{Y}_{2} \mathrm{O}_{3}$ undergoes a discontinuity of almost two orders of magnitude. In contrast, the mobility of undoped $\mathrm{Y}_{2} \mathrm{O}_{3}$ exhibits classical Arrhenius behavior.

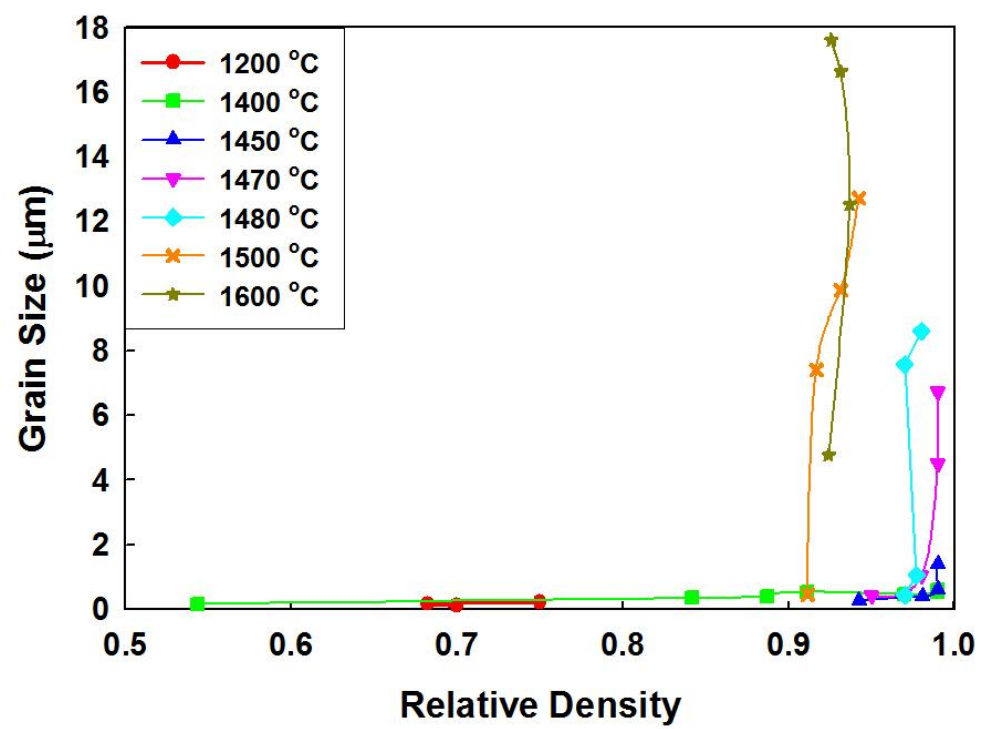

Figure 4: Grain size versus relative density trajectories for $10 \% \mathrm{Yb} / 1 \%$ Er-doped $\mathrm{Y}_{2} \mathrm{O}_{3}$ sintered at three different temperatures. At $1400{ }^{\circ} \mathrm{C}$, the trajectory is nearly flat, indicating densification without grain growth. At 1500 and $1600{ }^{\circ} \mathrm{C}$, the trajectory is nearly vertical, indicating grain growth without densification. 


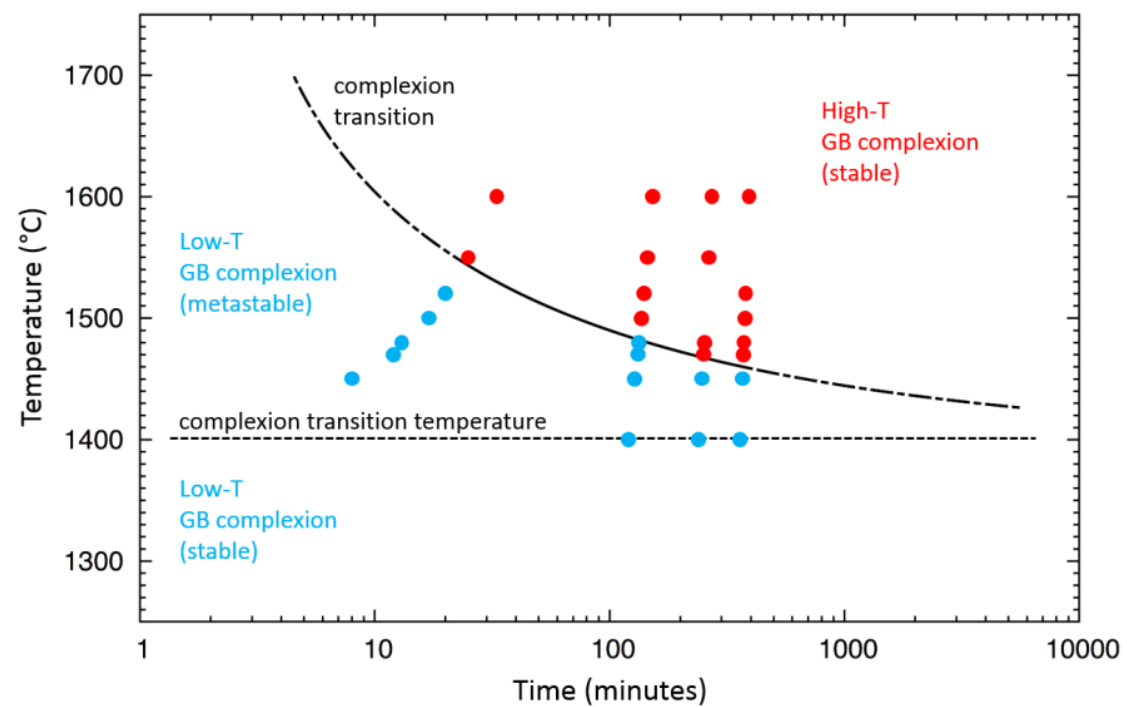

Figure 5: Grain boundary complexion TTT diagram for $10 \% \mathrm{Yb} / 1 \%$ Er-doped $\mathrm{Y}_{2} \mathrm{O}_{3}$ generated by plotting the grain growth data from Figure 2 on temperature-time axes. The blue circles represent points in timetemperature space at which small grains existed in the specimen (low temperature GB complexion is either stable or metastable). The red circles represent points after which the discontinuous jump in grain growth occurred resulting in very large grains (high temperature GB complexion is stable).
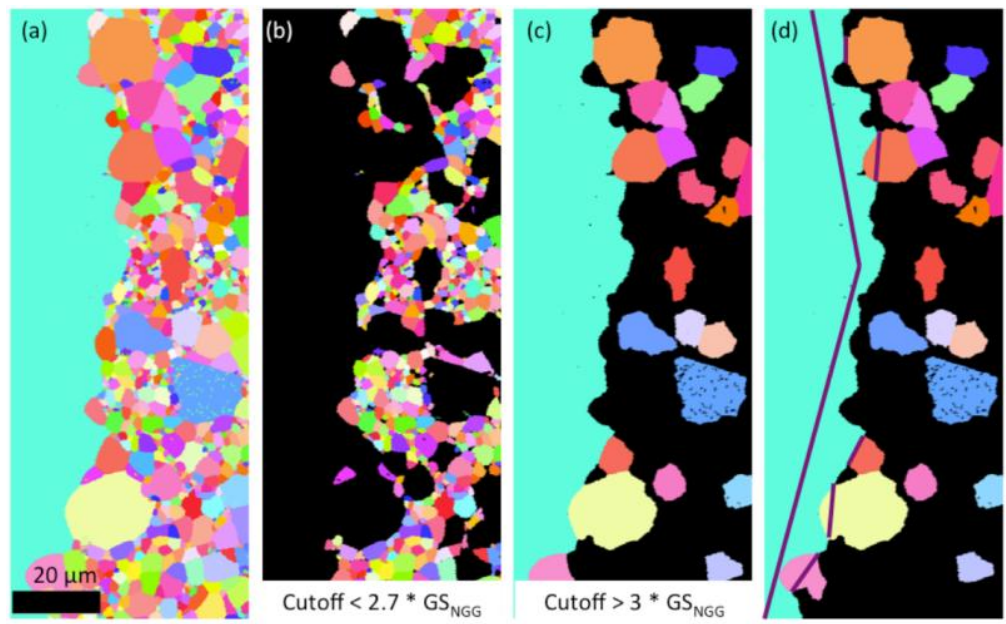

Figure 6: Method used to quantify the percentage of grain boundary transformed from the low temperature complexion to the high temperature complexion in $\mathrm{Y}$-doped $\mathrm{Al}_{2} \mathrm{O}_{3}$. (a) EBSD map of one of the single crystal interfaces; (b) A partition is set to filter out grains that are larger than 2.7 times the normal grain size; (c) The first partition is removed and a second partition is set such that all grains greater than 3 times the normal grain size are shown; (d) The total length of the single crystal interface and the length of the interface in contact with abnormal grains was measured. 


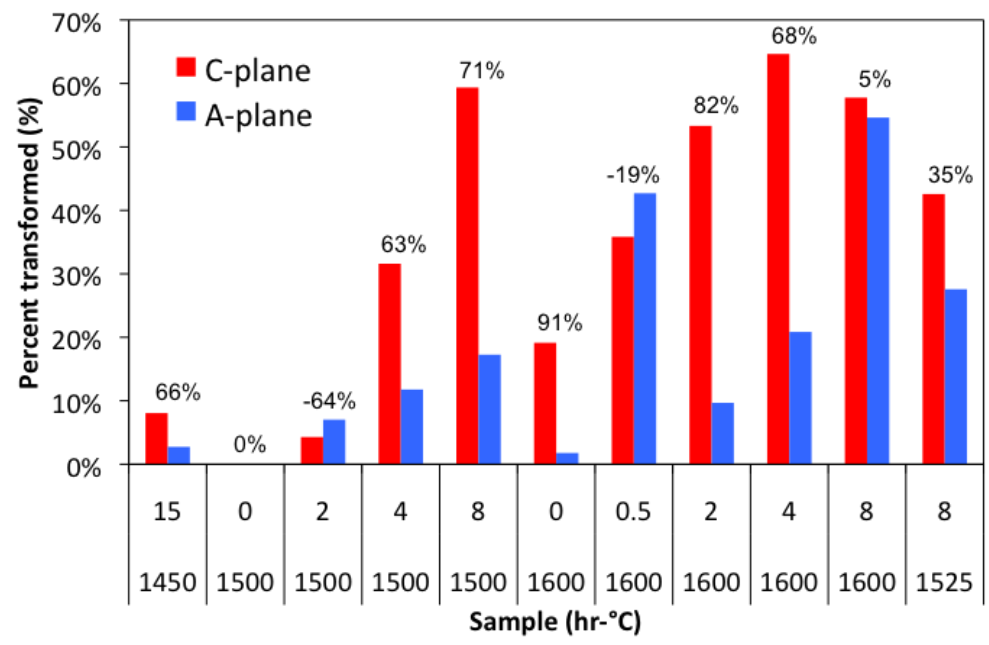

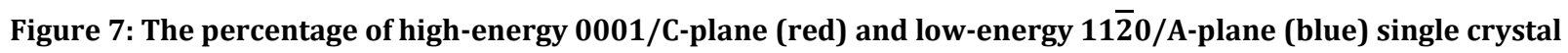
interfaces in contact with abnormal grains in $\mathrm{Y}$-doped $\mathrm{Al}_{2} \mathrm{O}_{3}$. Each pair of red and blue bars is a single annealing time and temperature combination. The numbers above the bars indicate the percentage difference between the amount of C-plane transformed and the amount of A-plane transformed.

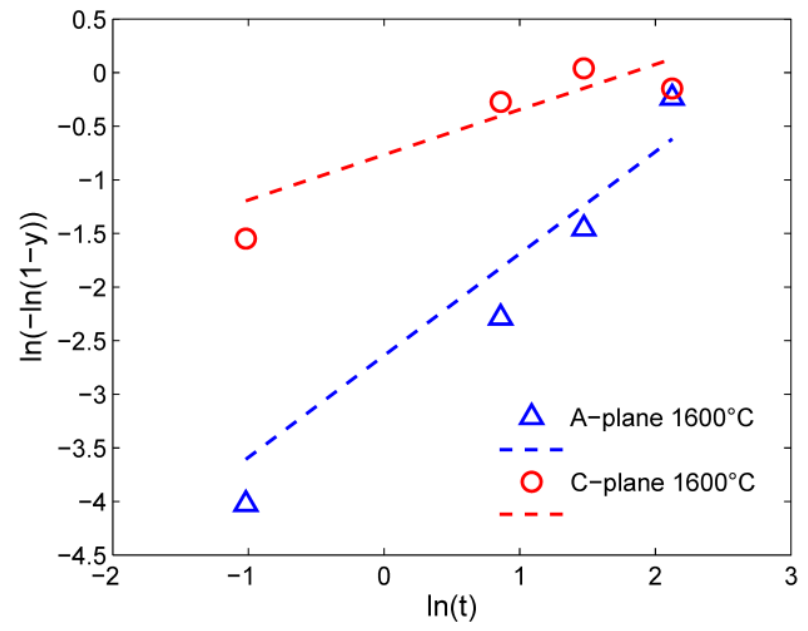

Figure 8: An illustration of the fitting process used to determine the Avrami parameters for complexion transitions at $1600{ }^{\circ} \mathrm{C}$. Best fit lines (dashed) are plotted according to Eq. (2), and their slope and intercept is used to determine the values of $n$ and $k$. 


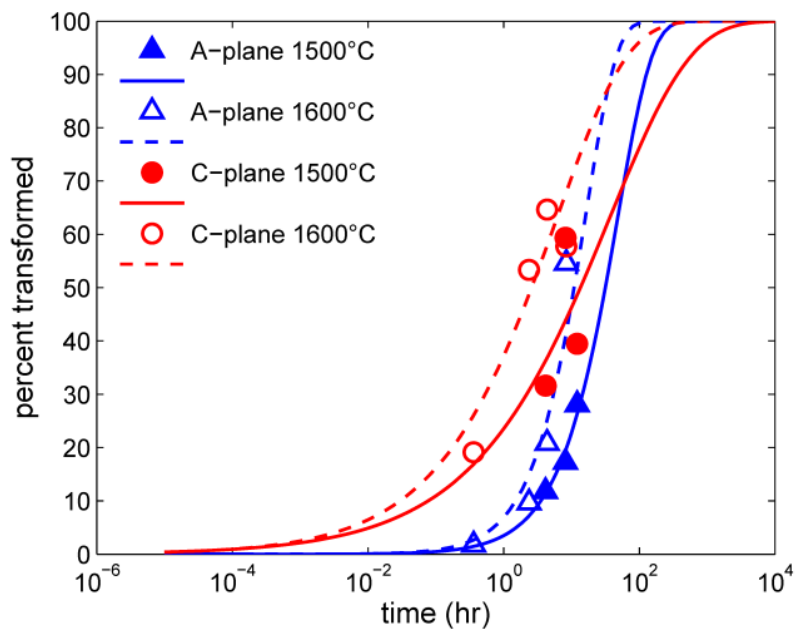

Figure 9: Grain boundary complexion transformation data from for $\mathrm{Y}$-doped $\mathrm{Al}_{2} \mathrm{O}_{3}$ plotted on a logarithmic time scale. Data points for the transformations along the A-plane (blue) and the C-plane (red) have been fitted with Avrami curves to show the complexion transition kinetics more clearly.

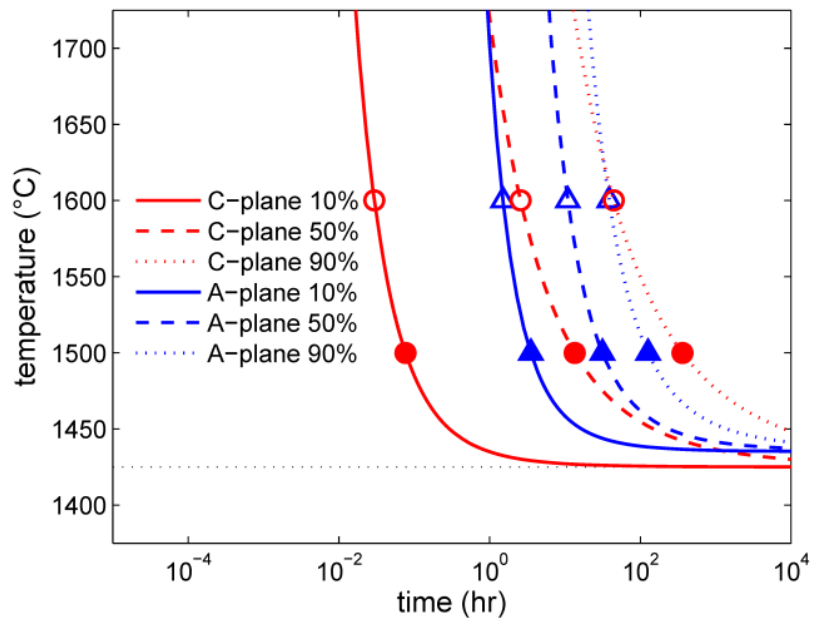

Figure 10: Grain boundary complexion TTT diagram for $\mathrm{Y}$-doped $\mathrm{Al}_{2} \mathrm{O}_{3}$, showing transformation curves for grain boundaries along the C-plane (red) and A-plane (blue) single crystal interfaces. Solid markers represent data points from fitted Avrami curves at $1500{ }^{\circ} \mathrm{C}$ while open markers represent data from fitted curves at $1600{ }^{\circ} \mathrm{C}$. 
Discontinuities in grain boundary mobility occur as a function of time, temperature, and crystallography...
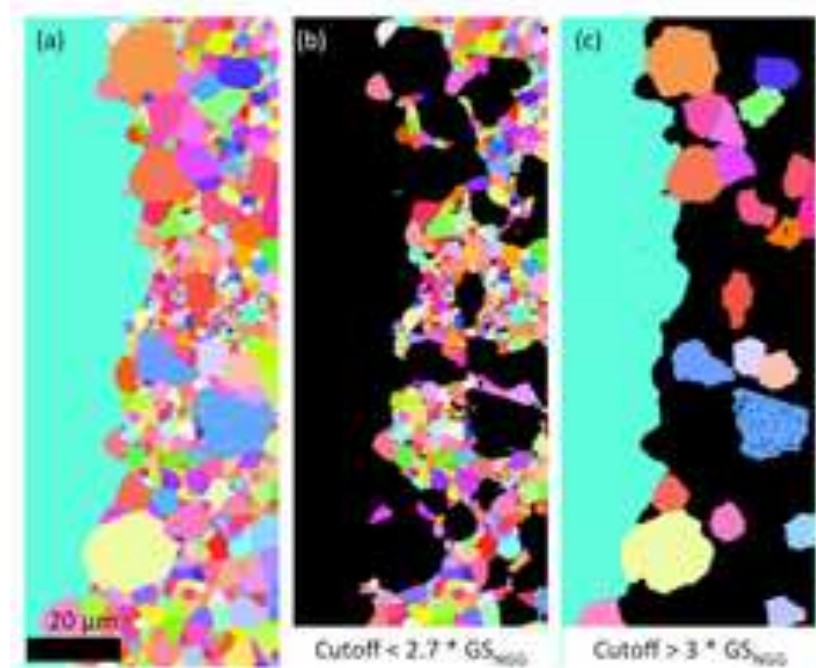

Cotoff $>3^{*}$ GS

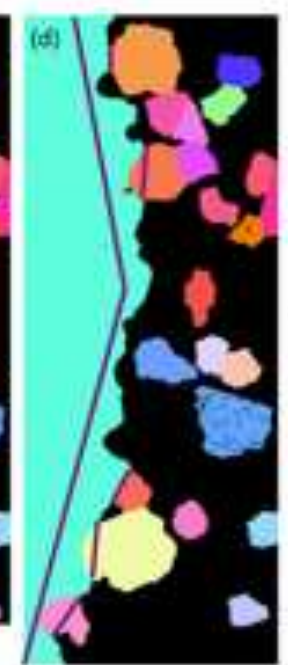

Time-Temperature-Transformation (TTT) Grain Boundary Complexion Diagram

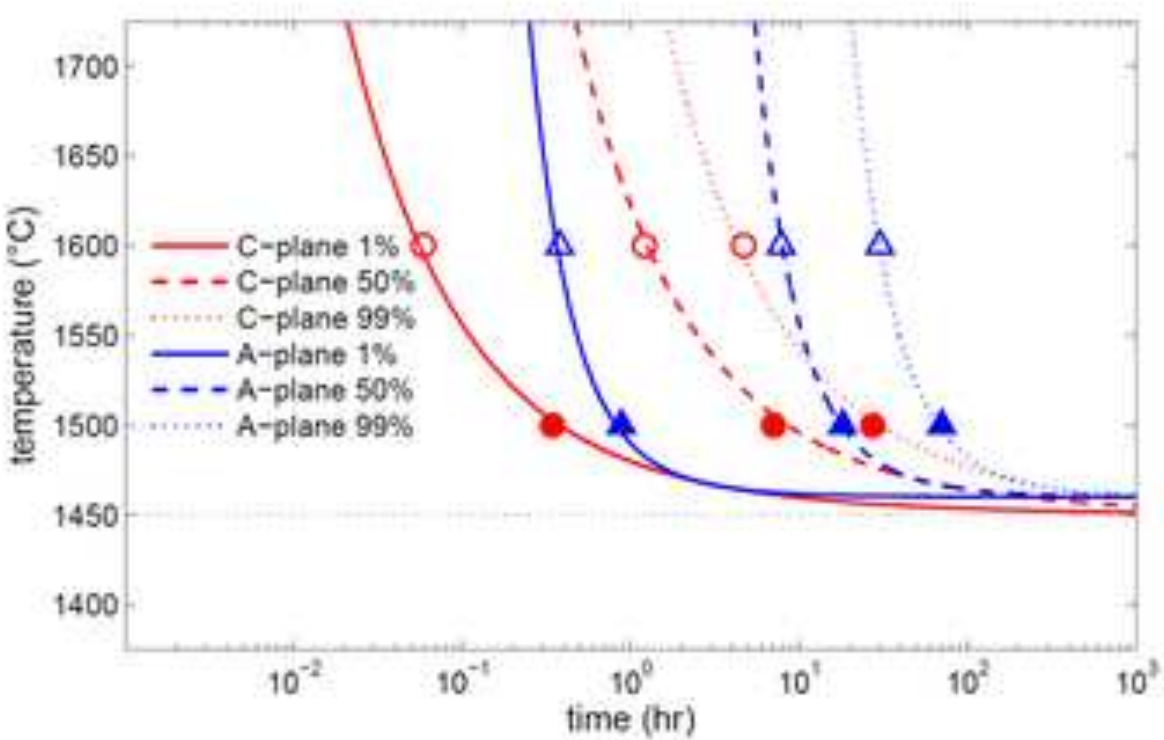

